\title{
ADAPTED SCALING FACTORS FOR DECODING CONCATENATED CODES BASED ON RS CODES
}

\author{
Es-said Azougaghe ${ }^{1}$, Abderrazak Farchane ${ }^{1}$, Said Safi ${ }^{1}$ and Mostafa Belkasmi ${ }^{2}$ \\ ${ }^{1}$ Laboratory of Mathematics Innovation and Information Technology (LIMATI), \\ Polydisciplinary Faculty, Sultan Moulay Slimane University \\ Beni Mellal, Morocco \\ ${ }^{2}$ Department of Communication Technology, Mohammed V Souissi University, \\ Rabat, National School of Computer Science and Systems Analysis (ENSIAS) \\ Rabat, Morocco \\ ${ }^{1}$ \{essaidazougaghe, a.farchane,safi.said \}@ gmail.com, \\ 23elkasmi@ensias.ma
}

\begin{abstract}
The performance of communication systems has been developed thanks to the application of the iterative decoding techniques. In this paper, a generalization of parallel concatenated block (GPCB) codes based on RS codes is presented. Simulations are used to look into the effect of different component codes, interleaver sizes and patterns, and the number of iterations. The simulation results show the relevance of the adapted parameters to decode generalized parallel concatenated block codes based on RS codes. The results obtained by using adapted parameters outperform those obtained with empiric parameters.
\end{abstract}

Keywords - RS codes, Chase decoding, Modified Chase-Pyndiah Algorithm, interleaver, Additive white Gaussian Noise (AWGN), Bit Error Rate (BER), Signal to Noise Ratio (SNR), iterative decoding, generalized parallel concatenated Block codes (GPCB)

\section{INTRODUCTION}

There are many reasons that have contributed to the massive interest in product codes. First of all, product codes have noticed a great growth as a result of the introduction of Turbo decoding [1]. In addition to this, the product codes are very identical to concatenated codes as well as to multilevel codes in the sense that almost any solution that works for product codes can easily be compatible with concatenated codes and multilevel codes. Many scholars have suggested different computation methods of soft value for iterative decoding of product codes. A case in example can be found in the works of Pyndiah et al. [2-5] who proposed a new iterative decoding algorithm based on Chase decoding [6-7]. The obtained results for product codes based on BCH codes suggested that there is a similarity with those obtained by convolutional turbo codes. Likewise, the generalized parallel concatenated block (GPCB) codes can be seen to be similar to convolutional turbo codes in both encoding and decoding structures. Iterative decoding of concatenated codes uses long powerful codes and keeps the decoder relatively simple. The length and power of these codes result in safety and durability of application.

Our study is based on RS codes that we decode by using the Modified Chase-Pyndiah algorithm(MCP)[8]. Our contribution, in this work, lies in that we tested the application of the Modified Chase-Pyndiah SISO algorithm to decode the GPCB-RS codes based on RS

Received: March 18, 2019

Reviewed: May 6, 2019

Accepted: May 10, 2019

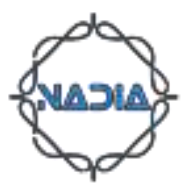


CODES, and we investigated the impact of various component codes, the number of iterations, interleaver size, length and pattern using simulations with an adapted scaling factor to the circumstances of the decoder, namely $\beta$ and $\alpha$.

Relevant studies adapted scaling factor to the circumstances of the decoder. The adapted parameter can outperform the previous empirical factor, except that the adapted parameter works without re-optimisation after every change in application. This can be noticed in the generalized serial concatenated block codes presented in [9] and parallel concatenated block codes in[10]. Unlike the aforementioned works that applied adapted scaling factor for BCH codes, our study applies this adapted parameter to decode GPCB-RS codes.

The residual part of this paper is organized as follow: Section 2 presents the encoder structure of the generalized parallel concatenated block codes. In Section 3, we present the component decoder. We describe the iterative decoding of the GPCB codes, in Section 4. The simulation results are given in Section 5. The last Section concludes this paper.

\section{GENERALIZED PARALLEL CONCATENATED BLOCK CODES (GPCB)}

\subsection{CONSTRUCTION}

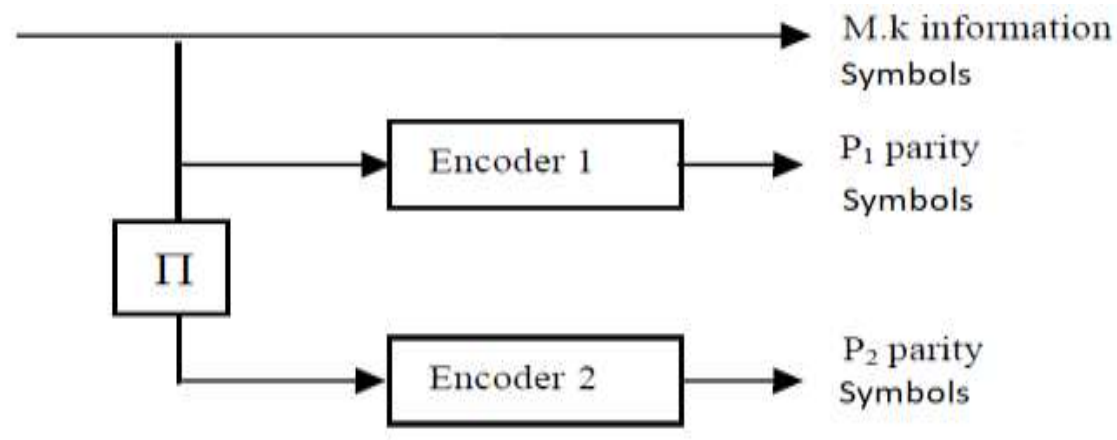

Fig. 1 Encoder Structure of GPCB

Figure 1 illustrates the construction of the generalized parallel concatenated block codes (GPCB). Here a block of $N=M . k$ data symbols at the input of the encoder is subdivided to $\mathrm{M}$ sub-blocks each of $k$ symbols. Each $k$ symbols vector is encoded in order to produce $\mathrm{n}$ symbols codeword. The input block is scrambled by the interleaver -denoted by $\Pi$ - before entering in the second encoder. The codeword of GPCB code, as shown in figure 2, consists of the input block followed by the parity check symbols of both encoders. In this contribution, several interleaving techniques were invoked such as random, helical, diagonal and primitive interleaver.

A systematic GPCB code is based on two component systematic block codes, $C_{1}$ with parameters $\left(n_{1}, k\right)$ and $C_{2}$ with parameters $\left(n_{2}, k\right)$. Viewing the coding scheme of 1 as single GPCB encoder, the length of the information-word to be encoded by the GPCB code is given by the size of the interleaver $N=M . k$. The first encoder produces $P_{1}=M \times$ $\left(n_{1}-k\right)$ parity check symbols. The second encoder produces $P_{2}=M \times\left(n_{2}-k\right)$ parity check symbols. Thus, the total number of parity symbols generated by the GPCB encoder is: $P=P_{1}+P_{2}=M \times\left(n_{1}+n_{2}-2 \times k\right)$. The length of the GPCB codeword is given by: $L=N+P=M \times\left(n_{1}+n_{2}-k\right)$. Consequently, the code rate of the GPCB codes can be computed by: $=\frac{N}{L}=\frac{k}{\left(n_{1}+n_{2}-k\right)}$. This implies that the GPCB code rate is independent of the interleaver size $N$. 


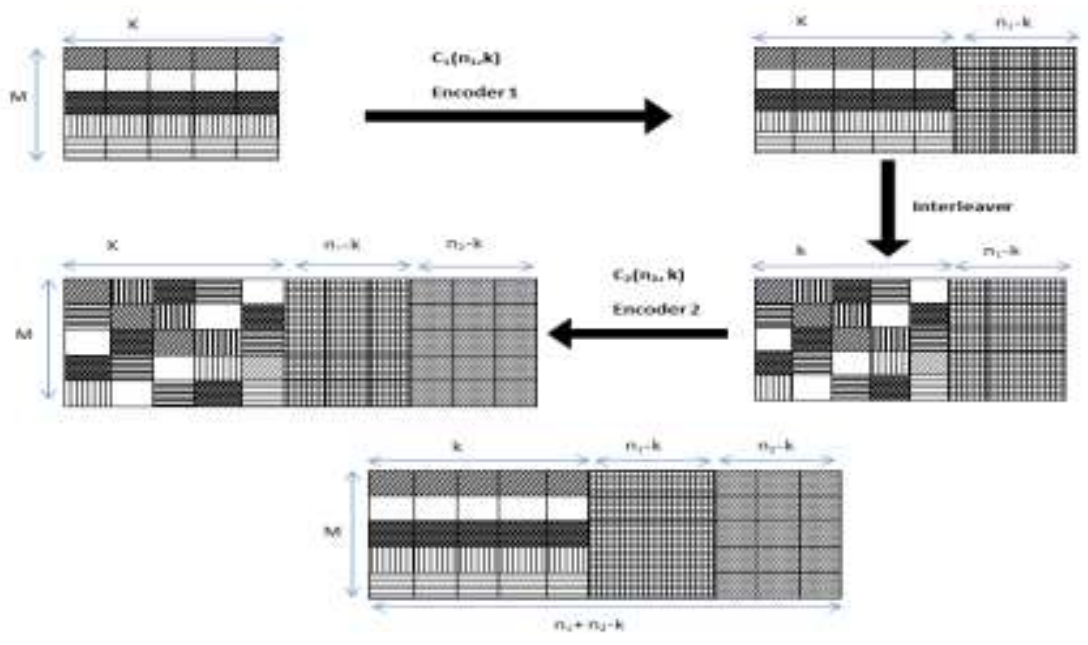

Fig. 2 Systematic GPCB encoding

\subsection{SOFT DECODING OF RS ODE}

If we consider the transmission of block coded binary symbols $\{-1,+1\}$ using BPSK signaling over a Gaussian channel, the sequence $\mathrm{R}$ at the input of the RS decoder has the following expression: $R=E+B$

where:

$$
R=\left(\begin{array}{lllll}
r_{11} & \cdots & r_{1 j} & \cdots & r_{1 n} \\
\vdots & \vdots & r_{11} & \vdots & \vdots \\
r_{m 1} & \cdots & r_{m j} & \cdots & r_{m n}
\end{array}\right)
$$

is the received sample word.

$$
E=\left(\begin{array}{lllll}
e_{11} & \cdots & e_{1 j} & \cdots & e_{1 n} \\
\vdots & \vdots & e_{11} & \vdots & \vdots \\
e_{m 1} & \cdots & e_{m j} & \cdots & e_{m n}
\end{array}\right)
$$

is the transmitted word.

$$
B=\left(\begin{array}{lllll}
b_{11} & \cdots & b_{1 j} & \cdots & n_{1 n} \\
\vdots & \vdots & r_{11} & \vdots & \vdots \\
b_{m 1} & \cdots & n_{m j} & \cdots & b_{m n}
\end{array}\right)
$$

are Additive White Gaussian Noise ( $A W G N$ ) samples of standard deviation $\sigma$. Decoding the received sequence $R$ according to the maximum likelihood criteria is given by:

$$
D=C^{i} \quad \text { if } \quad \operatorname{Pr}\left(E=C^{i} / R\right)>\operatorname{Pr}\left(E=C^{l} / R\right) \quad \forall l \neq i
$$

where:

$$
C^{i}=\left(\begin{array}{lllll}
C_{11}^{i} & \cdots & C_{1 j}^{i} & \cdots & C_{1 n}^{i} \\
\vdots & \vdots & C_{11}^{i} & \vdots & \vdots \\
C_{m 1}^{i} & \cdots & C_{m j}^{i} & \cdots & C_{m n}^{i}
\end{array}\right)
$$

is the $i^{\text {th }}$ code word of code $\mathrm{C}$ with parameters $(n, i)$ and 


$$
D=\left(\begin{array}{lllll}
d_{11} & \cdots & d_{1 j} & \cdots & d_{1 n} \\
\vdots & \vdots & d_{11} & \vdots & \vdots \\
d_{m 1} & \cdots & d_{m j} & \cdots & d_{m n}
\end{array}\right)
$$
to R.

The decision corresponding to maximum likelihood transmitted sequence conditionally

For received samples corrupted by AWGN, decoding rule (1) is simplified into : $D=C^{i}$ if $\left|R-C^{i}\right|^{2}<\left|R-C^{l}\right|^{2} \quad \forall l \neq i$ where:

$$
\left|R-C^{i}\right|^{2}=\sum_{j=1}^{n} \sum_{f=1}^{m}\left(r_{j f}-c_{j f}^{i}\right)^{2}
$$

\section{COMPONENT DECODER}

We choose as component decoder the Modified Chase-Pyndiah algorithm [8]. This decoder works as follows:

The decoder starts by generating a set of codewords which are in the vicinity of the received vector $R$. Then, among those codewords, it selects the nearest codeword from $R$ in term of Euclidean distance. By doing that it tries to determine the most likely codeword. The reliability of the decoded bits is given by the log likelihood ratio (LLR) of the decision $d_{i f}$ which is defined by:

$$
L L R_{i f}=\ln \frac{\operatorname{Pr}\left(e_{j f}=+1 / R\right)}{\operatorname{Pr}\left(e_{j f}=-1 / R\right)}
$$

Where $e_{j f}$ is the binary element in position $(j, f)$ of the transmitted code word E, $1 \leq$ $j \leq n$ et $1 \leq f \leq m$. The expression of the $L L R_{i f}$ can be approximated, in the case of the AWGN, by:

$$
L L R_{i f}=\frac{1}{2 \sigma^{2}}\left[\left|R-C^{\min (-1)}\right|^{2}-\left|R-C^{\min (+1)}\right|^{2}\right]
$$

Where $C^{\min (+1)}$ and $C^{\min (-1)}$ are two codewords at minimum Euclidean distance from $\mathrm{R}$ with $C_{j f}^{\min (+1)}=+1$ and $C_{j f}^{\min (-1)}=-1, C_{j f}^{\min (+1)}$ and $C_{j f}^{\min (-1)}$ are chosen among the subset of code word given by Chase algorithm. By expanding relation (2) we obtain:

$$
L L R_{i f}=\frac{2}{\sigma^{2}}\left(r_{j f}+\sum_{x=1 x \neq j}^{n} \sum_{z=1 z \neq f}^{n} r_{x z} c_{x z}^{\min (+1)} \rho_{x z}\right)
$$

Where

$$
\begin{gathered}
((x, z) \neq(j, f)) \\
\rho_{x z}=\left\{\begin{array}{lll}
0, & \text { if } & c_{x z}^{\min (+1)}=c_{x z}^{\min (-1)} \\
1, & \text { if } & c_{x z}^{\min (+1)} \neq c_{x z}^{\min (-1)}
\end{array}\right.
\end{gathered}
$$

If we normalize the approximated LLR of $d_{i f}$ with respect to $\frac{2}{\sigma^{2}}$ we obtain:

$$
r_{j f}^{\prime}=\left(\frac{\sigma^{2}}{2}\right) \cdot L L R_{i f}=r_{j f}+w_{j f}
$$

The estimated normalized LLR of decision $d_{i f}, r_{j f}^{\prime}$ is given by input samples $r_{j f}$ plus $w_{j f}$ which is independent of $r_{j f}$. The LLR of $r_{j f}^{\prime}$ is an estimation of the soft decision of the RS decoder.

To compute the normalized $L L R_{i f}$ of binary elements at the output RS decoder, we must first select the codeword at minimum Euclidean distance from R. Let $C^{\min (+i)}$ be this code word, $C^{\min (+i)}$ has a binary element $i$ at position $(j, f)(i= \pm 1)$. Then we look for codeword $C^{\min (+i)}$ at minimal Euclidean distance from $\mathrm{R}$ among the codeword subset obtained by Chase algorithm. 
$C^{\min (+i)}$ must have $-i$ as binary element at position $(j, f)$.

If the $C^{\min (+i)}$ codeword is found, the soft decision $r_{j f}^{\prime}$ can be computed using the relation given bellow:

$$
r_{j f}^{\prime}=\left(\frac{\left(M^{\min (-i)}-M^{\min (i)}\right)}{4}\right) c_{j f}^{\min (i)}
$$

Where $M^{\min (-i)}$ and $M^{\min (i)}$ represent respectively the $c_{j f}^{\min (-i)}$ Euclidean distance from R and $c_{j f}^{\min (i)}$ Euclidean distance from R.

Else we use the relation: $r_{j f}^{\prime}=\left(\frac{1}{2} \sigma_{R}+\left|r_{i j}\right|\right) c_{j f}^{\min (i)}$ where $\sigma_{R}$ is the standard deviation of the decoder input sequence $R$.

\section{ITERATIVE DECODING OF GPCB CODES}

\subsection{GPCB DECODER}

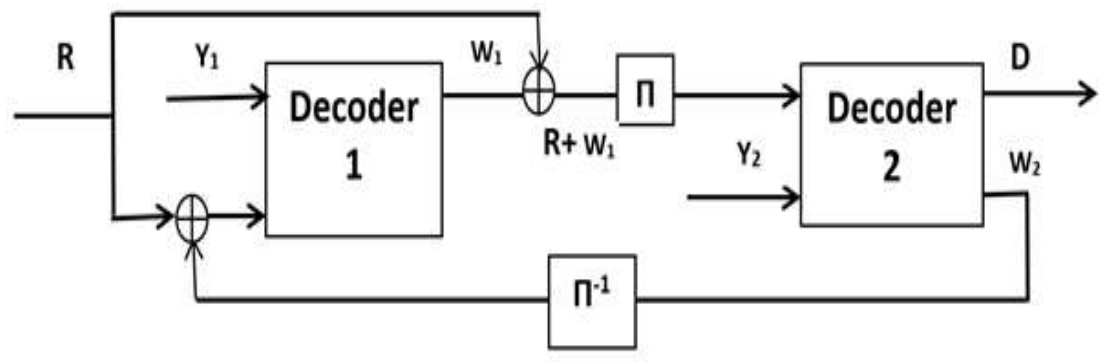

Fig. 3 Iterative decoding structure for the GPCB codes

The decoding of the GPCB codes is iterative. The decoder structure is shown in Figure 3. An iteration consists in using two component decoders serially. The first one uses the systematic information and the first parity check symbols in order to generate extrinsic information as in the Modified Chase-Pyndiah algorithm. This extrinsic information is used to update the reliabilities of the systematic information which will be interleaved and fed into the second decoder with the second parity check symbols received from the channel. The second decoder also generates the extrinsic information using Modified ChasePyndiah decoder, and then updates the reliabilities of the systematic information for the second time. The updated reliabilities will be desinterleaved and fed again into first decoder, for the next iteration. The process resumes until a maximum number of iterations is reached. The coefficients $\alpha$ and $\beta$ used in Chase-Pyndiah algorithm are listed in Table I.

Table II. Simulation Parameters

\begin{tabular}{|l|l|}
\hline Parameter & Value \\
\hline Modulation & BPSK \\
\hline Environment & The C Language \\
\hline Cannel & AWGN \\
\hline Interleaver & Random interleaver (default) \\
\cline { 2 - 2 } & Diagonal interleaver \\
\cline { 2 - 2 } & Primitive interleaver \\
\cline { 2 - 2 } & Helical interleaver \\
\hline $\boldsymbol{\alpha}$ & $0.00,0.01,0.08,0.12,0.16,0.20,0.24,0.28,0.32,0.36,0.40$, \\
\hline
\end{tabular}




\begin{tabular}{|l|l|}
\hline $\boldsymbol{\beta}$ & $0.56,0.60,0.64,0.68,0.72,0.76,0.80,0.82,0.86,0.88,0.90$, \\
& $0.91,0.93,0.95,0.97,0.99,0.99,1.00,1.00,1.00$ \\
\hline Elementary decoder & Modified Chase-Pyndiah, Chase-Pyndiah \\
\hline Iterations & from 1 to 20 (default) \\
\hline Interleaver size & $1 \times k, 10 \times k, 100 \times k, 300 \times k$ \\
\hline
\end{tabular}

\subsection{PARAMETERS $\alpha$ AND $\beta$}

We have determined the values of $\alpha$ and $\beta$ empirically [10]. These parameters play a crucial role to have good performance. So, the better parameters you have the better performance you will gain. Therefore, we should carefully determine these parameters. To obtain good parameters, we choose some condition for which codes are sensitive. Thus, we take the parameter $\mathrm{M}$ equal to 100 , and relatively high component code length.

We begin our process by setting the number of iterations in 1 , and we vary the value of $\alpha$, where $0<\alpha<1$, in order to have good performance, and keep the value of $\alpha$ which gives the best BER (bit error rate). Next, we vary the value of the parameter, where $0<$ $\beta<1$, in the same way.

Once the good parameters are chosen, for the first iteration, we increment the number of iterations, and we look for the good ones for the second iteration. Then we come back without decrementing the number of iterations so as to adjust the parameters $\alpha$ and $\beta$ for eventual improvement of the performance. Afterwards, we increment the number of iterations and keep repeating the same process until a maximal number of iterations is reached.

\subsection{ADAPTED PARAMETER $\alpha(p)$}

The role of the parameter $\alpha(p)$ is vital in the decoding performance. In the works [3] [10] [11] [2] [11], this parameter was experimentally predetermined. Its values are chosen such as the $B E R=10^{-5}$ is attained with the minimum number of iterations. This process is costly in terms of time and hardware. We have adapted the parameters to the circumstances of the product codes and turbo like-codes to overcome this problem. The following formula gives the expression of $(p)$ :

$$
\alpha(p)=\frac{1}{\sigma_{W(p-1)}^{2}}
$$

where $\sigma_{W(p-1)}^{2}$ denote the variance of the extrinsic information delivered by the previous decoder. The performance obtained by using the adapted parameter $\alpha(p)$ is comparable to those obtained by the predetermined parameter. Therefore, we don't need to re-optimize this parameter if we change the application.

\section{RESULTS AND DISCUSSION}

In this section, the performances of generalized parallel concatenated block codes based on RS codes are evaluated. Transmission over the additive white Gaussian noise (AWGN) in channel and binary antipodal modulation are used. We are interested in the information bit error rate (BER) for different signal to noise ratios per information bit $\frac{E_{b}}{N_{0}}$ in $\mathrm{dB}$. There are many parameters which affect the performance of GPCB-RS codes when decoded with iterative decoder. Accordingly, we studied the effects of the following parameters on the decoder performance, namely the number of decoding iterations, the component codes and interleaver size and patterns. 


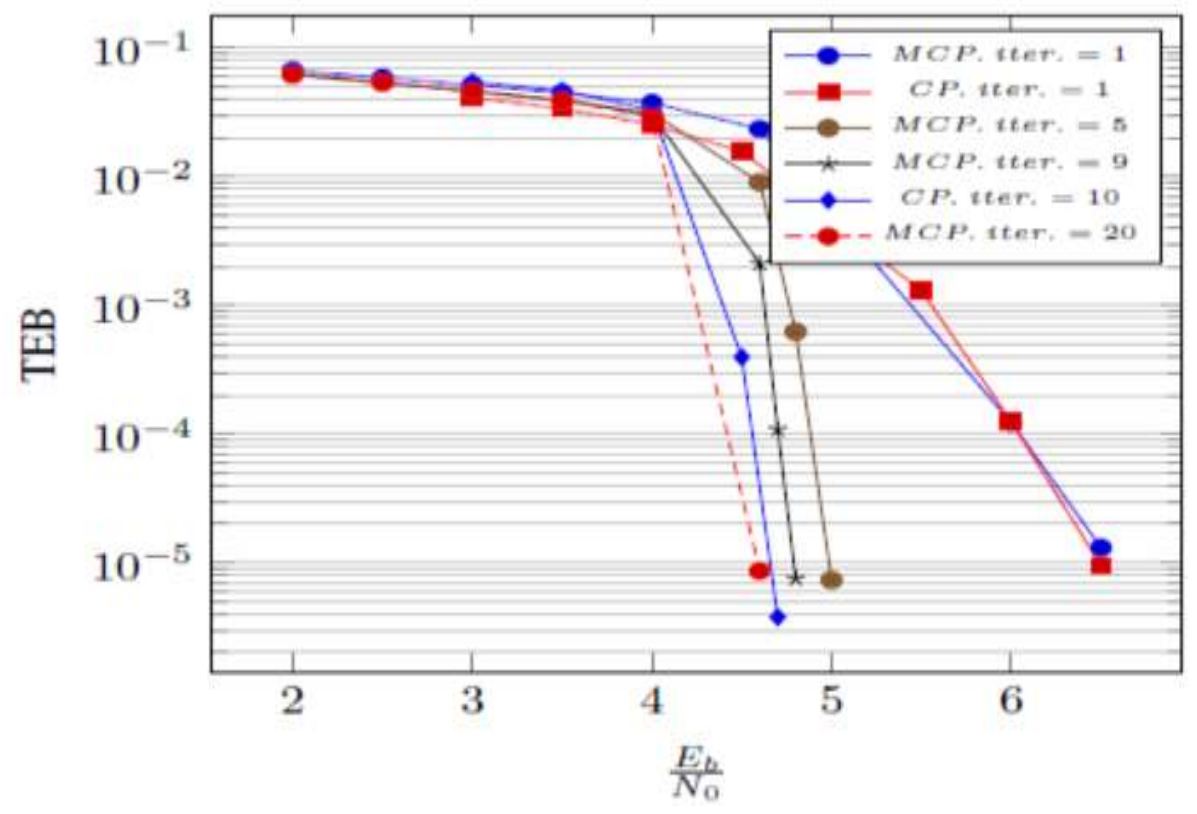

Fig. 4 Effect of iterations on iterative decoding of GPCB-RS $(67,59)$ Code, with $\mathrm{M}=100$, over AWGN channel

Figure 4 shows the performance of the code GPCB-RS $(67,59)$, with $M=100$. This figure shows that the slope of curves and coding gain are improved by increasing the number of iterations. After the $10^{\text {th }}$ iteration, the amelioration of the coding gain becomes negligible for Chase-Pyndiah decoder (CP), whereas the Modified Chase-Pyndiah decoder (MCP) can go up to the $20^{\text {th }}$ iteration. This makes our paper a significant contribution in this field.

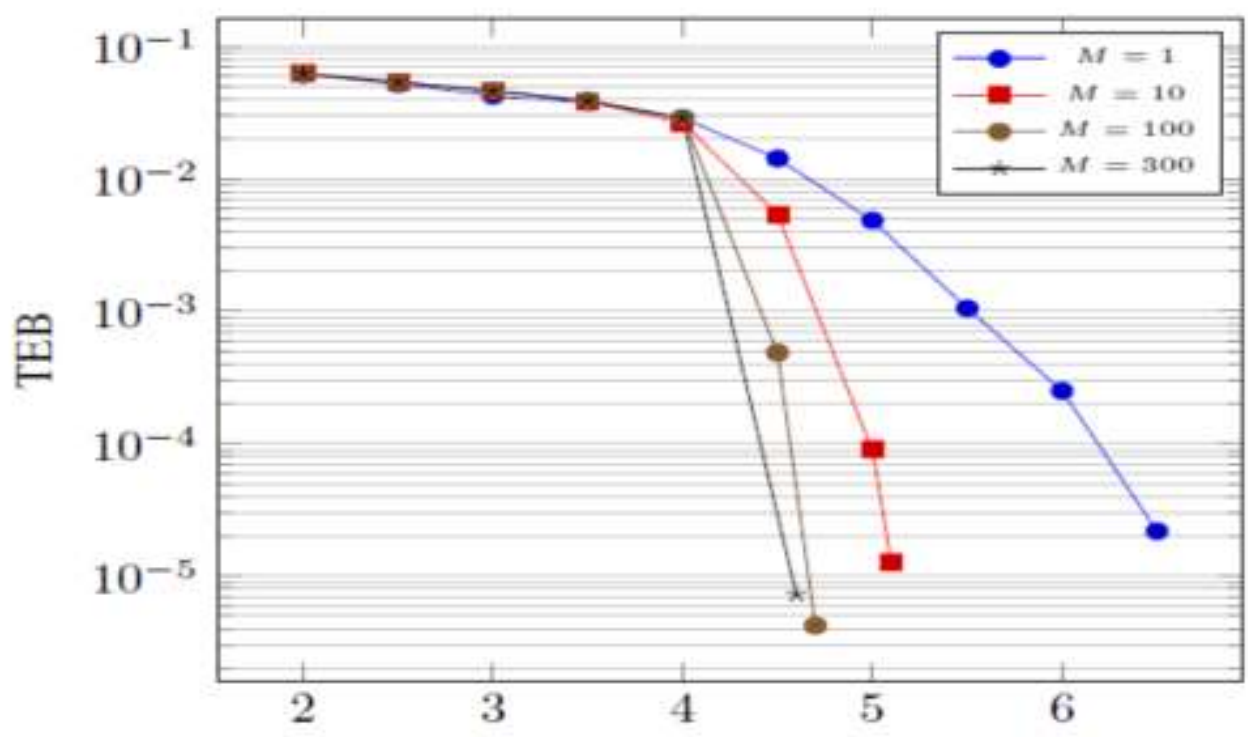

Fig. 5 Effect of the parameter M on iterative decoding of GPCB-RS(67, 59) code, using MCP decoder, over AWGN channel

Figure 5 shows the effect of the multi-block $M$. The gain reaches $1.4 \mathrm{~dB}$ as we pass from $M=1$ to $M=10$, decreases to $0.4 \mathrm{~dB}$ between $\mathrm{M}=10$ to $\mathrm{M}=100$ and becomes negligible beyond. This demonstrates how effective is the multi-block. 


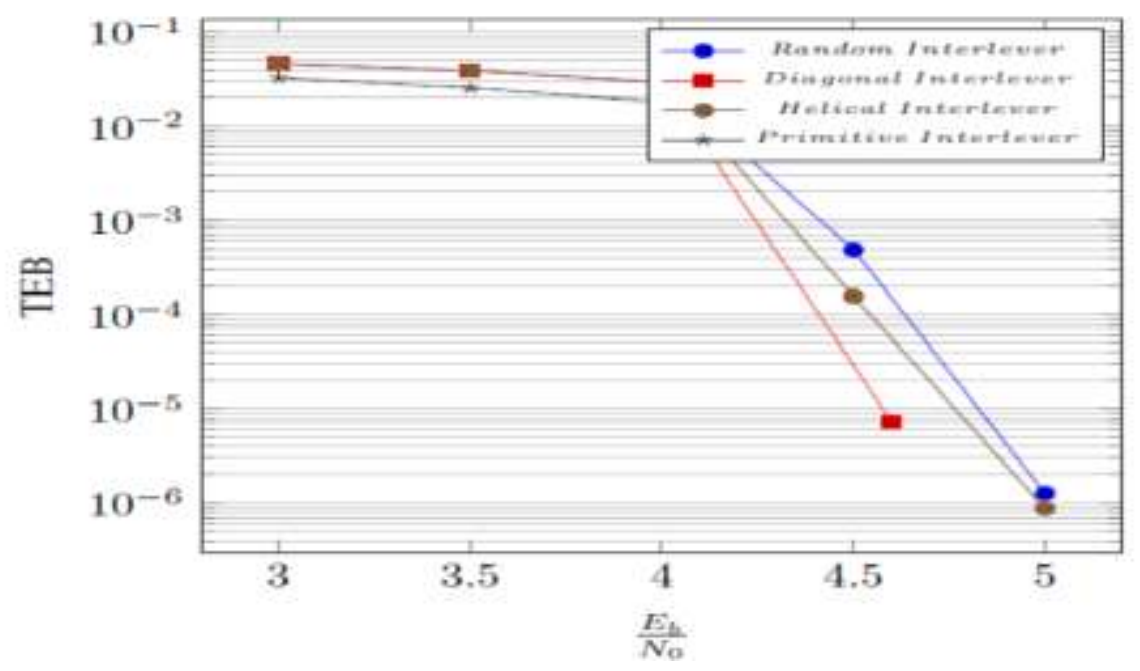

Fig. 6 Interleaver structure effect on Iterative decoding of GPCB-RS(67, 59) Code, with $\mathrm{M}=100$, over AWGN channel

To study the influence of the interleaver pattern on the GPCB-RS codes performance, we have evaluated the BER versus $\frac{E_{b}}{N_{0}}$ of the GPCB-RS $(67,59)$ code using different interleaver structures such as diagonal, helical, primitive and random interleaver with parameter $\mathrm{M}=100$.

Figure 6 depicts the performance results. According to this figure, we observe that the random interleaver outperforms the other ones by about $0.5 \mathrm{~dB}$ at $\mathrm{TEB}=10^{-5}$.

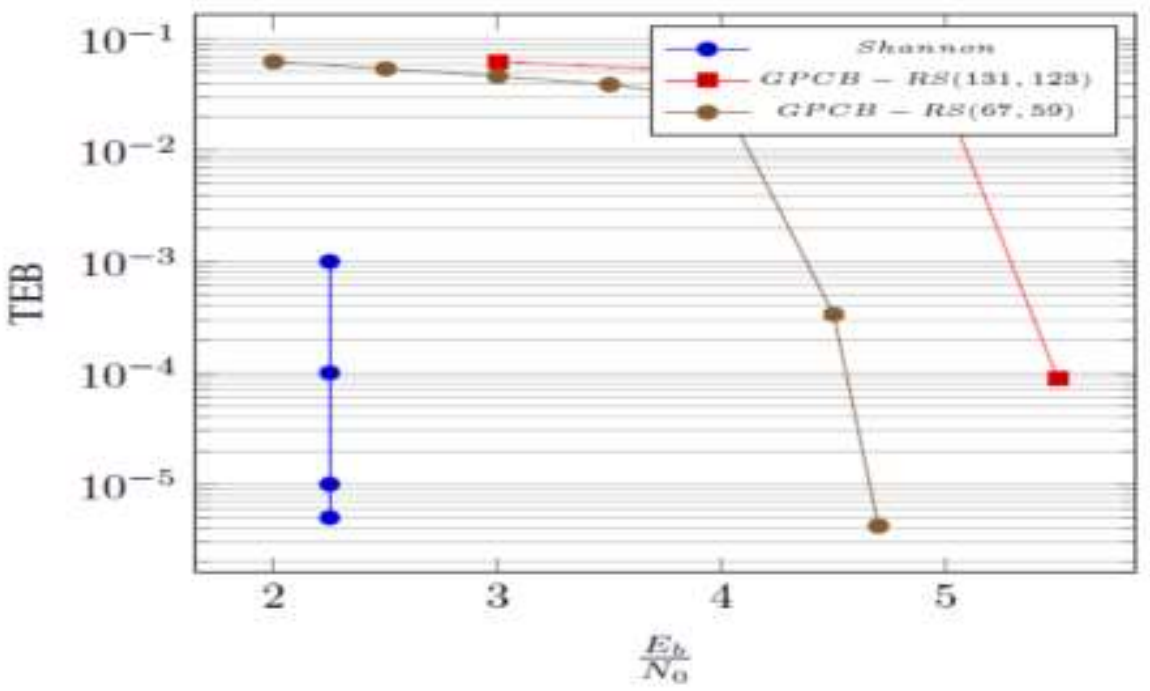

Fig. 7 Performance comparison between GPCB-RS $(69,57)$, GPCB-RS $(131,123)$ over AWGN channel

To evaluate the performance of the generalized parallel concatenated block codes, we compare the coding gain at the $20^{\text {th }}$ iteration of the following codes GPCB-RS $(67,59)$, GPCB-RS $(131,123)$, with the same code rate 0.82 and the parameter $M=100$. The performance is shown in Figure 7. From this figure, we observe that the performance becomes worse with increasing the length of the component code. The GPCB-RS $(69,57)$, GPCB-RS $(131,123)$ codes are respectively 2.3 and 2.8 away from their Shannon limits. 


\section{CONCLUSIONS}

In this paper, we have extended the work that has been done to decode generalized concatenated block codes based on BCH codes for RS codes. We have used adapted parameters in order to avoid determining its value empirically. The simulation results show that the adapted parameters are effective, as it can be demonstrated in the asymptotic performance.

The obtained results look very promising and open new perspectives. In this regard, this work can be extended to decode generalized serially concatenated block based on RS codes, by using adapted parameters.

\section{REFERENCES}

[1] Q. Zhai and Y. Wang, "Stochastic resonance in parallel concatenated turbo code decoding", Digit. Signal Process., vol. 56, p. 93-99, sept. 2016.

[2] A. Picart and R. Pyndiah, "Adapted iterative decoding of product codes", in Global Telecommunications Conference, 1999. GLOBECOM'99, 1999, vol. 5, p. 2357-2362.

[3] O. Aitsab and R. Pyndiah, "Performance of concatenated Reed-Solomon/convolutional codes with iterative decoding", in Global Telecommunications Conference, 1997. GLOBECOM'97., IEEE, 1997, vol. 2, p. 934-938.

[4] Seok-Ho Chang, P. C. Cosman and L. B. Milstein, "Iterative Channel Decoding of FEC-Based MultipleDescription Codes", IEEE Trans. Image Process., vol. 21, nº 3, p. 1138-1152, mars 2012.

[5] J. Son, J. J. Kong and K. Yang, "Efficient decoding of block turbo codes", J. Commun. Netw., vol. 20, ${ }^{\circ}$ 4, p. 345-353, août 2018.

[6] D. Chase, "Class of algorithms for decoding block codes with channel measurement information", IEEE Trans. Inf. Theory, vol. 18, $\mathrm{n}^{\circ}$ 1, p. 170-182, 1972.

[7] P. Wu and N. Jindal, "Performance of Hybrid-ARQ in Block-Fading Channels: A Fixed Outage Probability Analysis", ArXiv08114191 Cs Math, nov. 2008.

[8] A. Farchane and M. Belkasmi, "New efficient decoder for product and concatenated block codes", J. Telecommun., vol. 12, p. 17-22, 2012

[9] A. Farchane and M. Belkasmi, "Generalized serially concatenated codes: construction and iterative decoding", Int. J. Math. Comput. Sci., vol. 6, nº 2, 2010.

[10] Abderrazzak Farchane, Mostafa Belkasmi and N. Said, "Generalized parallel concatenated block codes based on BCH and RS codes, construction and Iterative decoding", ArXiv Prepr. ArXiv13034224, 2013.

[11] M. Belkasmi and A. Farchane, "Iterative decoding of parallel concatenated block codes", in Computer and Communication Engineering, 2008. ICCCE 2008. International Conference on, 2008, p. 230-235. 
International Journal of Advanced Science and Technology Vol.126 (2019) 